\title{
INSPECTION OF A MEDIEVAL WOOD SCULPTURE USING COMPUTER TOMOGRAPHY
}

\author{
K. Kapitany, A. Somogyi, A. Barsi \\ Budapest University of Technology and Economics (BME), Dept. of Photogrammetry and Geoinformatics, Hungary - \\ (kapitany.kristof, somogyi.arpad, barsi.arpad)@epito.bme.hu
}

Commission V, WG V/2

KEY WORDS: computed tomography, wood sculpture analysis, 3D modelling

\begin{abstract}
:
Computer tomography (CT) is an excellent technique for obtaining accurate 3D information about the human body. It allows to visualize the organs, bones and blood vessels, furthermore it enables to diagnose anomalies and diseases. Its spatial reconstruction capability supports other interesting applications, such as inspecting different, even valuable objects like ancient sculptures. Current paper presents a methodology of evaluating CT and video imagery through the example of investigating a wood Madonna with infant Jesus sculpture from the $14^{\text {th }}$ century. The developed techniques extract the outer boundary of the statue, which has been triangulated to derive the surface model. The interior of the sculpture has also been revealed: the iron bolts and rivets as well as the woodworm holes can be mapped. By merging the interior and outer data (geometry and texture) interesting visualizations (perspective views, sections etc.) have been created.
\end{abstract}

\section{INTRODUCTION}

The paper was intended to document a research where the processing of expensive computer tomography (CT) and low-cost mobile camera imagery were combined. The purpose of the research is to acquire as many information as possible about a medieval wood sculpture. The object of the research is a wood Madonna sculpture owned by the Hungarian Museum of Fine Arts, Budapest. The result is intended not only to create a documentation of a single art treasure, but to elaborate a technology for similar objects using also some new techniques, like spatial segmentation, data fusion and $3 \mathrm{D}$ printing.

\section{THE MADONNA (BY A MASTER FROM FABRIANO)}

The sculpture is of the second half of the $14^{\text {th }}$ century made supposedly by an unknown master from Fabriano, Italy. The height, width and depth of the painted wood sculpture is $153 \mathrm{~cm}, 48 \mathrm{~cm}$ and $38 \mathrm{~cm}$ respectively. The Madonna holds the child Jesus in her hand (Fig. 1), which is removable to ease the clothing at big religious ceremonies. On the backside of the Madonna there is a quadratic 20 by $15 \mathrm{~cm}$ niche with silk and velvet lining, and a door with ornamental lock. The niche has dealt presumably for keeping valuable relic. The sculpture was carved from a single wood $\log$ with a terminal board of size 74 by $8 \mathrm{~cm}$. The infant Jesus was carved also from a single log of wood. The arms were fitted later.

The face of the Madonna shows an archaic style, but a restoration in 2008-2009 has proven that it was earlier redesigned: the ornamental crown was removed, the rim of the kerchief was partly trimmed. Some fingers were failing, partly complemented. The arms of the infant Jesus were failing, their completion was ugly, rapid intervention made by glue, nails and also leather pieces. The sculpture was affected by strong insect offense. These injuries were corrected during the accurate and professional restoration and the sculpture has become now a beautiful art treasure of the Museum. (HMFA, 2009)

* Corresponding author

\section{DATA ACQUISITION: FILM AND COMPUTER TOMOGRAPHY}

The data acquisition was twofold: The Madonna was captured by a simple mobile phone camera (Samsung Galaxy S5) taken full HD film frames having a geometric resolution of $1920 \times 1080$ pixels. The camera is equipped by a 16-megapixel sensor and $\mathrm{f} / 2.2$ lens with an equivalent focal length of $31 \mathrm{~mm}$. The camera was rotated several times around the head of the Madonna, so two films were captured with a length of 34 and 31 seconds. The films have together 1944 frames. The mp4 format films can be loaded and split into independent frames. The later processing steps get every $10^{\text {th }}$ frame (sampling). The four films were taken from different height relatively to the Madonna head, so a better geometric reconstruction can be achieved. The frames were processed by Agisoft PhotoScan (see Section 4).

The CT tomograms were made in the laboratory of the Materials Science Department of Széchenyi István University with an YXILON CT Modular industrial CT-system (MGC41), with a resolution of $0.3 \mathrm{~mm}$. Originally the $\mathrm{CT}$ technique was used for medical analysis. X-ray is attenuated as it goes through different materials and textures. The capability of X-ray absorption can be characterized by the coefficient of X-ray absorption. If the energy transfer is constant, the absorption of X-ray depends only on the material through which it goes. This degraded radiation reaching the detectors generates electrical signals. There is a relationship between CT relative density and density of different minerals. On the CT images the radiodensity of a voxel can be quantified according to the Hounsfield scale that is depending on the average linear attenuation coefficient of the particular material in reference of distilled water on standard pressure and temperature.

Fig. 2 presents the wood sculpture during the CT imaging procedure. The only preparation necessary for CT scanning is to ensure that the object fits inside the field of view and that it does not move during the scan (Ketcham and Carlson, 2001). The wood sculpture was placed on a sample-holder, which rotates the object during the imaging. The infant was scanned in two parts to acquire the current resolution.

Fig. 3 presents a CT slice from the upper body of the infant. On 


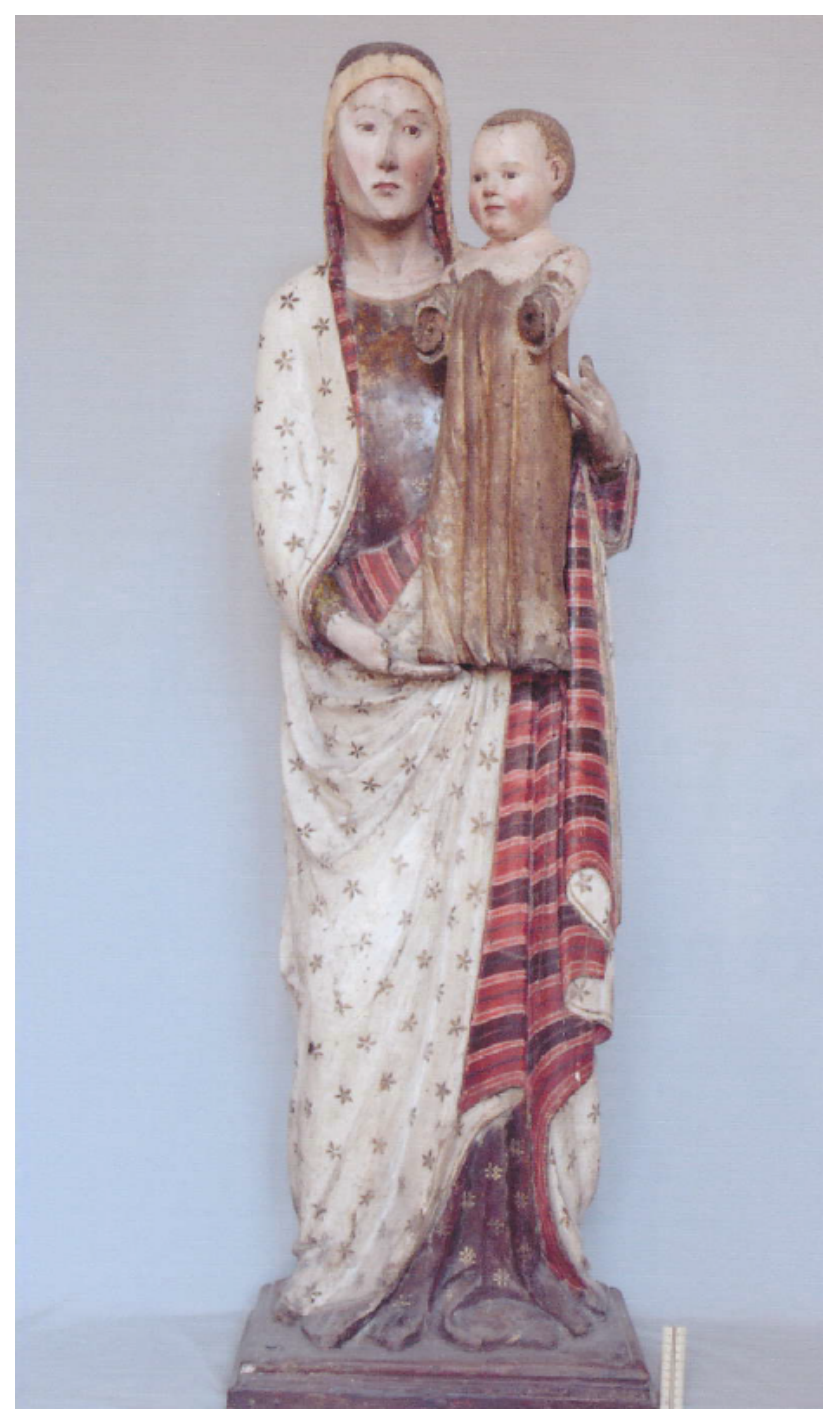

Figure 1: Sculpture of Madonna with child Jesus before the restoration (HMFA, 2009)

this image the different materials can be easily distinguished. The arms of the infants were cut off and replaced during the restoration, and the different materials with the imperfect joints could be seen on the images. There is a narrow gap between the cut surface and the new arms, and also the cross-section of a rivet that fixes the prosthesis to the body could be seen. The material of the wood is also interesting; because of the density differences, the growth-rings of the wood with the cross-sections of the woodworm holes are also visible.

The lower part of the Madonna sculpture was also imaged by CT. Fig. 4 presents the overview of the CT images where the different components (e.g. metal nails, rivets, the reliquary with its lock) are clearly visible.

\section{EVALUATION OF THE FILMS AND PHOTOS}

In the past few years there was a rapid development in the techniques of the image based modeling (IBM), especially in the structure from motion (SFM) and dense image matching methods. Due to these improvements the passive sensors (cameras) are more often applied to capture reality based 3D models. The images can be taken either with a static camera (still shots) or a moving camera (video sequence). Video imaging is much easier

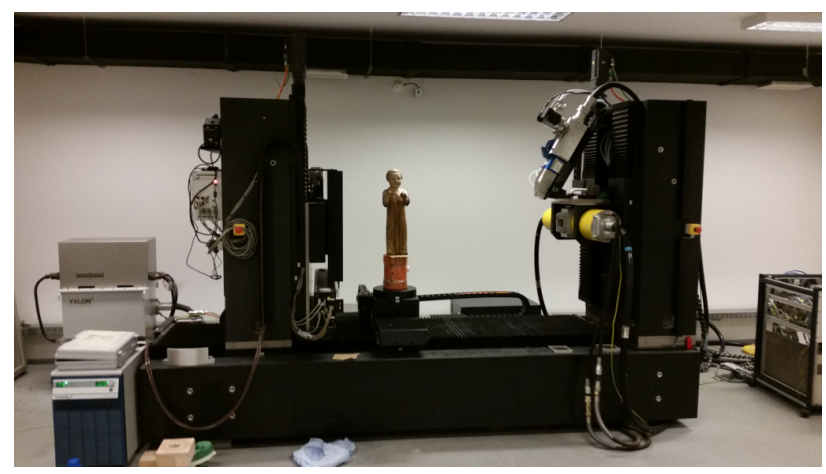

Figure 2: CT imaging of the wood sculpture

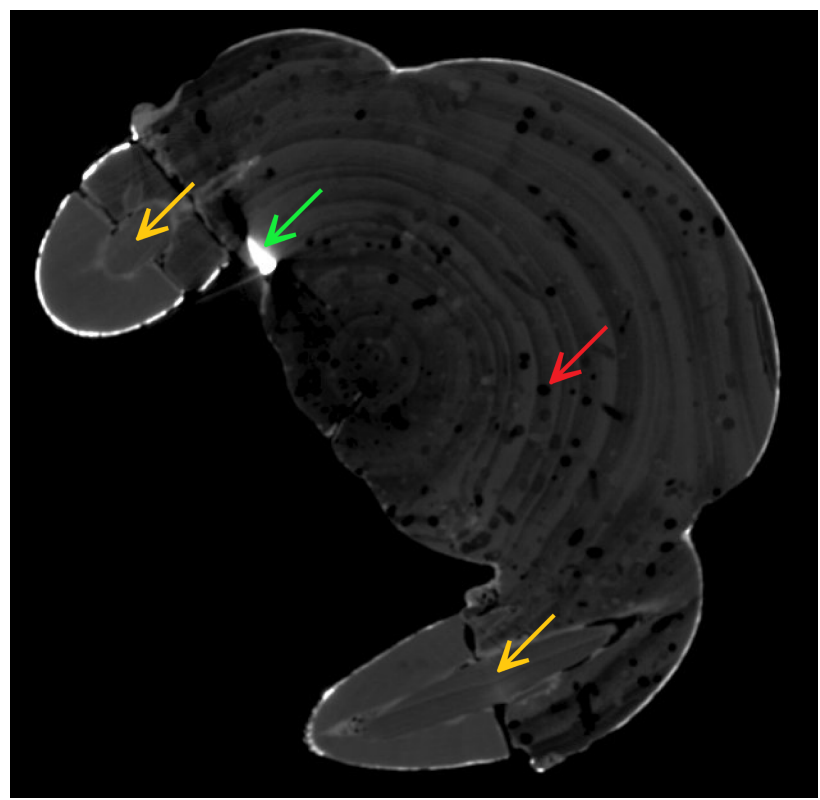

Figure 3: A CT image from the upper body of the infant. On the image the cross-section of the rivet (green arrow), woodworm holes (red arrow), and wood joints (yellow arrows) are marked

to apply when compared to still image shooting because the latter requires the proper positioning of the cameras during the capture, and to ensure the required overlapping of the images. However, there are three problems when video image sequences are used for highly detailed modeling. These problems are: the lower resolution of video images, the need of more computational capacity and blur effects due to camera shake on a significant number of images. But if a less accurate model is appropriate then the results would be satisfying. (Alsadik et al., 2015)

Both commercial (e.g. Agisoft PhotoScan or Pix4D Mapper Enterprise) and open-source development software (e.g. VisualSFM) are available for the object reconstruction. Our model was created by Agisoft PhotoScan (Table 1). The video was captured by a Samsung Galaxy S5 smart phone in two video segments. At first, image frames were extracted from the recorded videos. An improvement of the raw frame based model was necessary, because there were holes, "flying material drops" and other reconstruction errors (Fig. 5 and Fig. 6). The correction was performed by Geomagic Studio. The results could be shared or published as 3D Pdf (Fig. 7) (web.fmt.bme.hu/fmtpontfelho/madonna.pdf).

To recreate the sculpture into an interactive tangible format, 3D printing is an appropriate option. For that it was necessary to have a model with a lower polygon number but without the loss of de- 


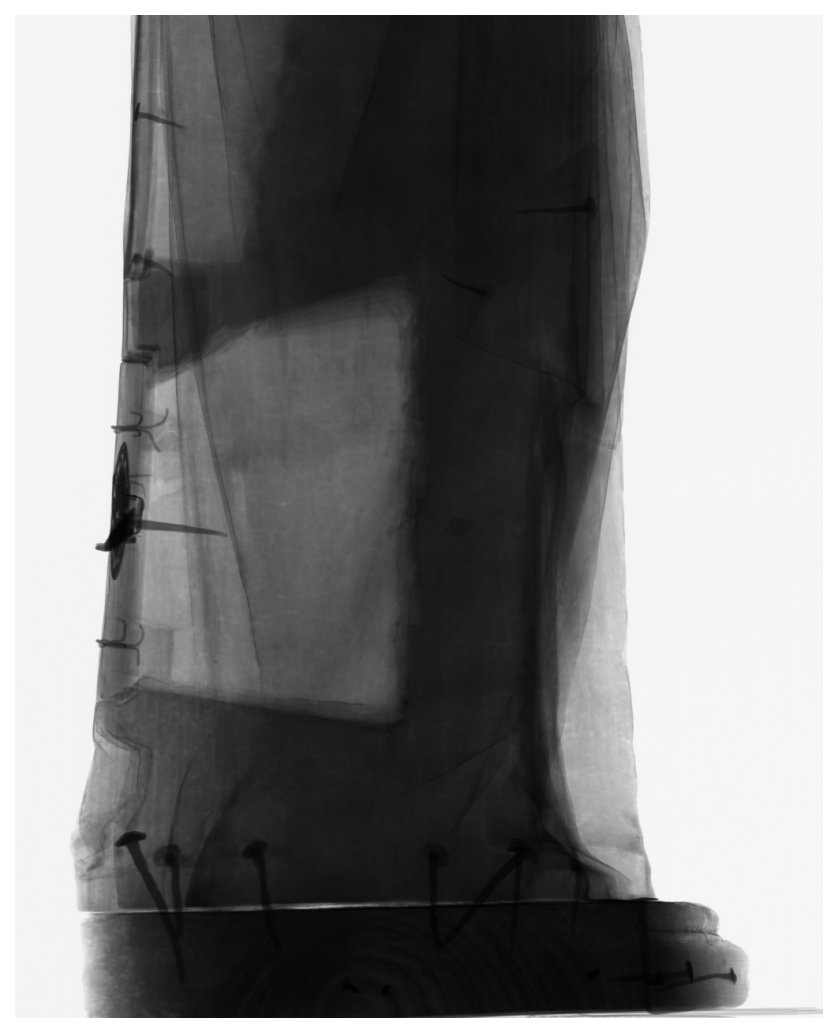

Figure 4: Overview of CT images, reliquary of the Madonna sculpture

tail. The resampling was also done in Geomagic Studio. Fig. 8 presents the 3D printed plastic model.

\section{EVALUATION OF THE CT IMAGERY}

The CT slices were processed by automated algorithms in Matlab environment using predefined parameters without any user interaction to acquire the outer surface of the sculpture and the woodworm holes. To segment the rivets and wood joints after the automated process, some artifacts were removed manually too. The segmentation of the wood sculpture, the bolts and rivets, and the woodworm holes was performed with thresholding (Russ, 2002), but additional filters and corrections were necessary to reduce noise and beam hardening effects (Buzug, 2004), (Van de Casteele, 2004). The algorithms process the CT slices successively one at a time. The result of the segmentation is a series of binary images, which could be further analyzed. The coordinates of the boundary-pixels were extracted from the images created a point cloud, where the image plane was used as the horizontal plane, and slice serial numbers were used for the vertical axis. The nails, rivets and the wooden joint composed three individual datasets. The mentioned point clouds formed the basis of the mesh model, performed by Geomagic Studio (Fig. 9). The reconstruction of the inner tubular surfaces (i.e. woodworm holes) is a complicated task for the mesh-modeling software. These could be visualized as point clouds, or as volume rendering as Fig. 10 presents the woodworm holes using the open-source Java based software, ImageJ. (ImageJ, 2016) The sculpture could be decomposed (Fig. 11)

\begin{tabular}{|l|l|l|}
\hline Image set & Aligned image count & 1804 \\
\cline { 2 - 3 } & Image resolution & $1080 \times 1920$ \\
\hline \multirow{3}{*}{$\begin{array}{l}\text { Sparse point } \\
\text { cloud }\end{array}$} & Point number & 39079 \\
& & \\
\cline { 2 - 3 } & $\begin{array}{l}\text { Matching and align- } \\
\text { ment time* }\end{array}$ & $\begin{array}{l}14 \mathrm{~h} 47 \mathrm{~min} \text { and } \\
1 \mathrm{~h} 23 \mathrm{~min}\end{array}$ \\
\cline { 2 - 3 } & Optimization time & $10 \mathrm{~min}$ \\
\hline \multirow{2}{*}{$\begin{array}{l}\text { Dense point } \\
\text { cloud }\end{array}$} & Point number & 2667782 \\
\cline { 2 - 3 } & Processing time & $9 \mathrm{~h} \mathrm{38} \mathrm{min}$ \\
\hline \multirow{3}{*}{ 3D model } & Faces of model & 533556 \\
\cline { 2 - 3 } & Processing time & $2 \mathrm{~min}$ \\
\cline { 2 - 3 } & Texturing time & $2 \mathrm{~min}$ \\
\hline
\end{tabular}

${ }^{*}$ High accuracy, generic pair preselection, 40000 key- and 1000 tie points limit

**High quality, depth filtering

Table 1: Data about the imagery and models

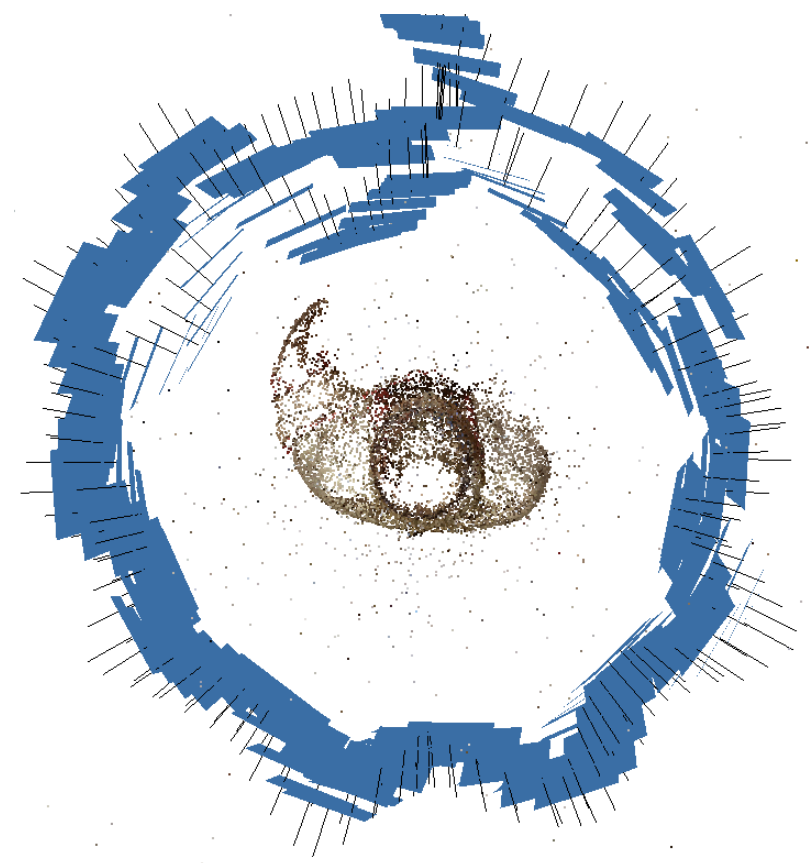

Figure 5: The aligned image frames (top view)

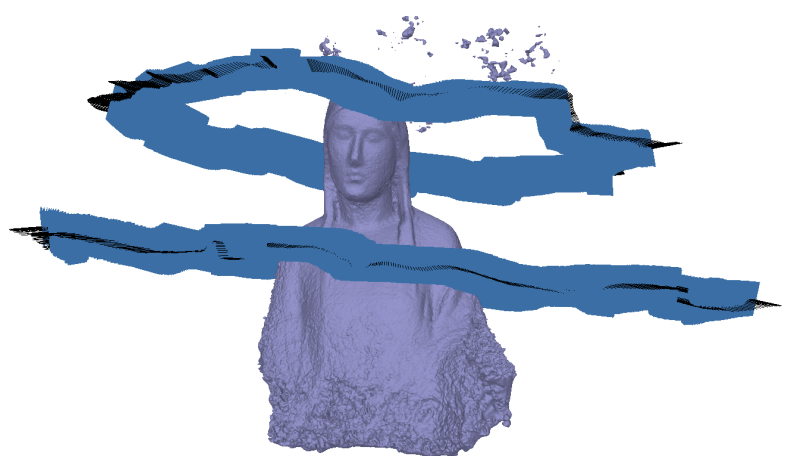

Figure 6: The two separated video trajectories and the small "flying drops" around the statue

\section{CONCLUSION}

The pilot research has proven that two distinct inspection technique can smoothly be joined for performing broad analyses of 


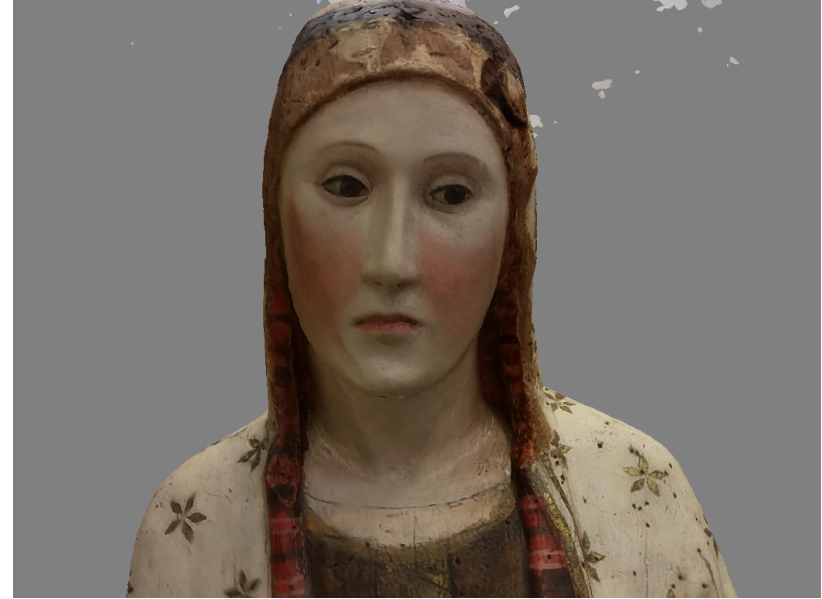

Figure 7: Textured image based model

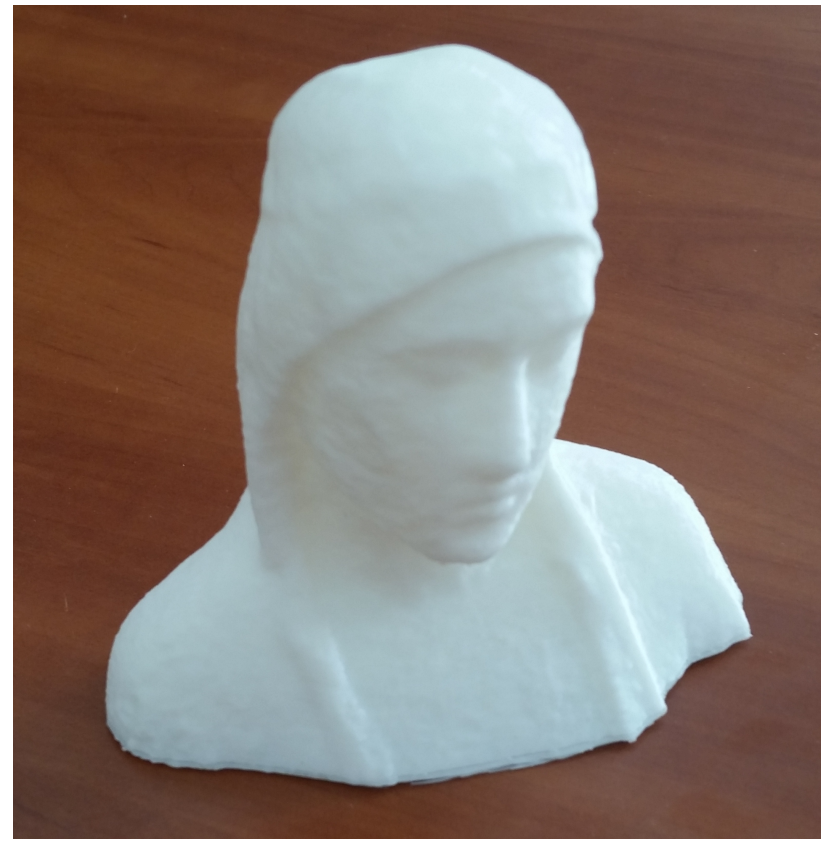

Figure 8: 3D printed plastic model

valuable medieval sculptures. The built-in cameras of the mobile phones mean low-cost and fast data acquisition technique, even for museum staff members. The obtained full-HD videos have great geometric resolution making them suitable for the use of the semi-global matching (SGM) based dense 3D object reconstruction. The potential output of the technique is not only a virtual object model, but is an adequate data source for nowadays 3D printers. The technology has become already mature for capturing also small details.

The other technology is the expensive but highly potential computed tomography, which requires unique and quite voluminous equipment, as well as trained operators. The ordinary use of CT is medical: it helps to diagnose for doctors (radiologists) in a non-invasive manner. Other application of the technology is in the material sciences, where for example invisible failures can be detected without any samplings or cuttings. In the meaning of the latter use we also wanted to obtain information about the inner status of the sculpture. Air contained holes, like wood worm holes, but also "strange" material inlays can also be detected, like nails, prostheses or other elements. The CT based reconstruction

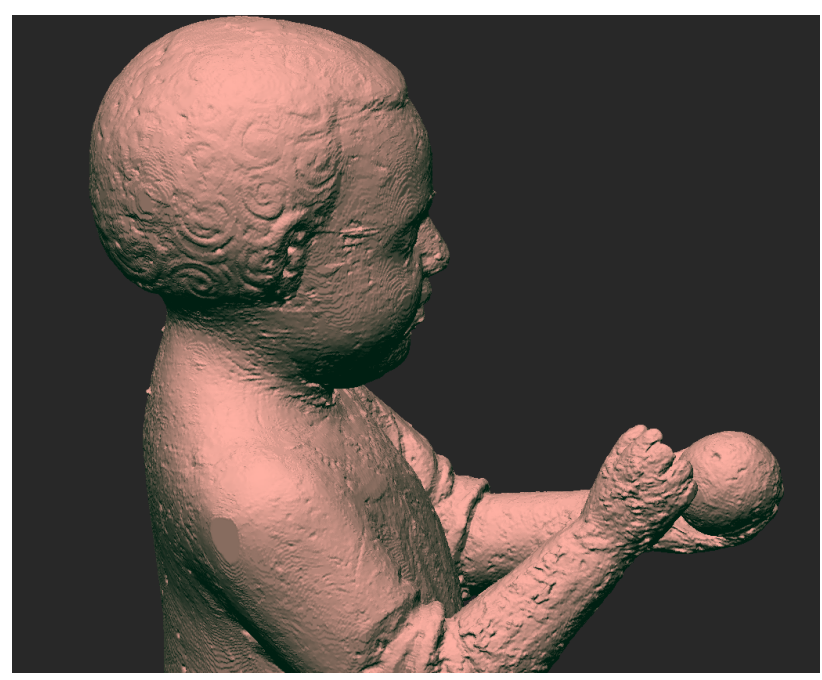

Figure 9: 3D surface of the infant based on the CT images

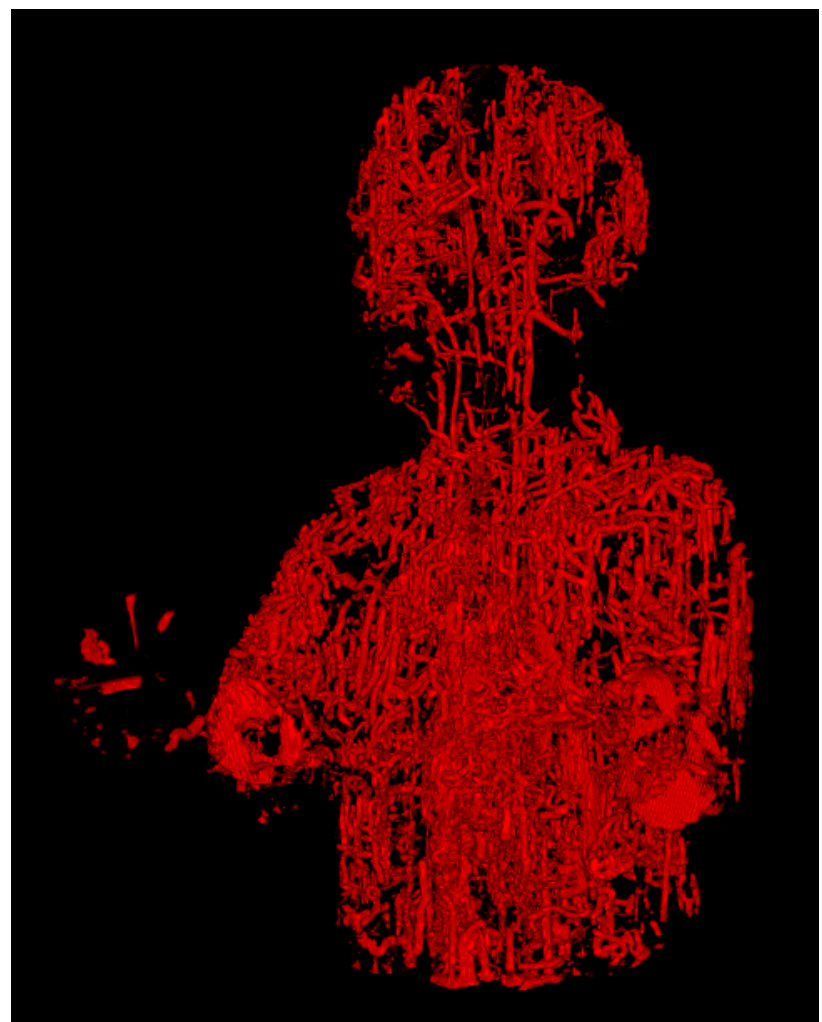

Figure 10: Wood worm holes in the upper body of the infant

is capable to derive the outer surface of the sculpture, which has excellent amount and density of points for joining to the model built from the exteriorly captured information.

The detailed analyses of the wood worm holes can be a base to elaborate a maintenance method to keep the sculpture in a healthy state. Other potential seems in the very fine differentiation of the $\mathrm{X}$-rayed material segments: the wood types have different microstructure (compactness, amount and direction of wood fibers etc.) which can be taken as features for recognizing the wood components of a sculpture. This step can be helpful for checking the originality of ancient art treasures. 


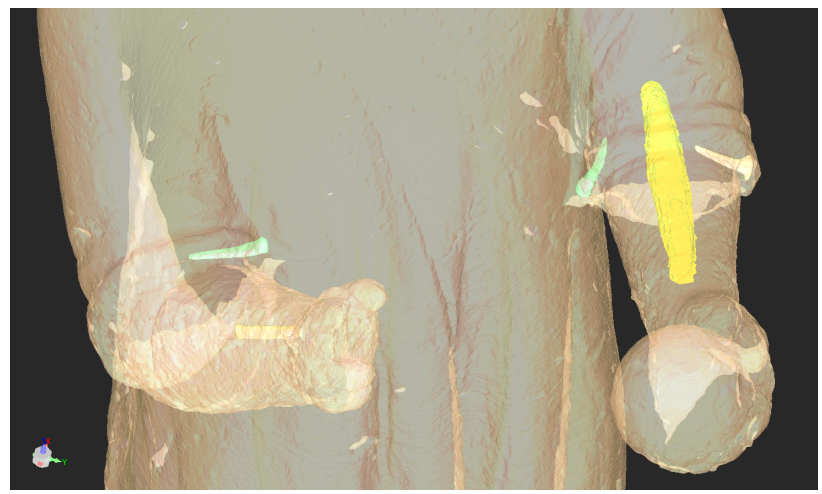

Figure 11: 3D object reconstruction of the infant. Nails and rivets beside the wooden joint (in one hand) are highlighted

\section{ACKNOWLEDGEMENTS}

We thank our colleagues from Hungarian Museum of Fine Arts, Budapest for preparing and transporting the sculpture and Széchenyi István University, Győr for performing the necessary CT imaging.

\section{REFERENCES}

Alsadik, B., Gerke, M. and Vosselman, G., 2015. Efficient use of video for $3 \mathrm{~d}$ modelling of cultural heritage objects. ISPRS Ann. Photogramm. Remote Sens. Spatial Inf. Sci. II-3/W4, pp. 1-8.

Buzug, T. M., 2004. Einfuhrung in die Computertomographie. Springer Verlag.

HMFA, 2009. Restoring documentation of the madonna with infant jesus - unpublished report. Hungarian Museum of Fine Arts, Budapest p. 21

ImageJ, 2016. Imagej software for digital image processing. http://imagej.nih.gov/ij/index.html.

Ketcham, R. and Carlson, W., 2001. Acquisition, optimization and interpretation of $\mathrm{x}$-ray computed tomographic imagery: applications to the geosciences. Computers \& Geosciences 27(4), pp. 381-400.

Russ, J. C., 2002. Image Processing Handbook, Fourth Edition. 4th edn, CRC Press, Inc., Boca Raton, FL, USA.

Van de Casteele, E., 2004. Model-based approach for beam hardening correction and resolution measurements in microtomography. Antwerpen. 